\title{
reCHERches
}

Culture et histoire dans l'espace roman

4 | 2010

Les représentations du corps dans la litterature latinoaméricaine

\section{Le corps-cosmos de la voix poématique dans Las palabras del cuerpo, de Lourdes Espínola}

\section{Dominique Casimiro}

\section{(2) OpenEdition}

\section{Journals}

Édition électronique

URL : https://journals.openedition.org/cher/8142

DOI : $10.4000 /$ cher.8142

ISSN : 2803-5992

Éditeur

Presses universitaires de Strasbourg

Édition imprimée

Date de publication : 30 juin 2010

Pagination : 245-260

ISBN : 978-2-35410-007-0

ISSN : 1968-035X

Référence électronique

Dominique Casimiro, «Le corps-cosmos de la voix poématique dans Las palabras del cuerpo, de Lourdes Espínola », reCHERches [En ligne], 4 | 2010, mis en ligne le 15 décembre 2021, consulté le 26 janvier 2022. URL : http://journals.openedition.org/cher/8142 ; DOI : https://doi.org/10.4000/cher. 8142

Ce(tte) œuvre est mise à disposition selon les termes de la Licence Creative Commons Attribution Pas d'Utilisation Commerciale - Partage dans les Mêmes Conditions 4.0 International. 


\section{Le corps-cosmos de la voix poématique dans Las palabras del cuerpo, de Lourdes Espínola \\ DOMINIQUE CASIMIRO \\ Université de Paris III - Sorbonne Nouvelle / Universidad de Chile}

$\mathrm{V}$

ers 1990, après 35 années de dictature, surgit sur la scène éditoriale paraguayenne un courant d'écriture poétique féminine totalement transgresseur. En effet, c'est après la disparition du dictateur que se produit la fin symbolique de la rhétorique du patriarcat qui avait lésé en particulier les droits et les libertés des femmes. Cette nouvelle expression féminine, chez un groupe hétérogène de femmes écrivaines, va ainsi refuser l'image sempiternelle de la mère très aimante et entièrement dévouée à ses enfants. Des femmes vont alors fustiger les institutions, le cléricalisme et, de façon générale, les clichés sur ce que doivent être les aspirations des femmes. Après plus de trente ans de corsetage, le corps féminin aspirait en toute logique à se libérer et à s'épanouir.

Transgressant tabous et interdits, Lourdes Espínola entendait elle aussi faire résonner, en toute liberté, sa voix. Non pour mener un combat égalitariste, revendicatif ou vindicatif, mais, pour assumer l'écriture comme un acte de combat en tant que création, production, opposées au rôle biologique et culturel de la femme-mère-reproductrice. Le but pour Lourdes Espínola est que les voix des femmes ancestrales ne se taisent plus («Las voces de mujeres ancestrales / que callaron su boca / tapadas por las manos de tu gente» Lourdes Espínola 2001 : 108) et que résonne la voix de la liberté retrouvée. Écoutons, par exemple, le poème suivant, intitulée «De cómo escriben las mujeres poemas eróticos», où le sujet lyrique ironise sur la 
censure dont étaient autrefois victimes celles qui avaient le malheur d'être des femmes et qui, comble de la provocation et de l'impudeur, osaient écrire des poèmes érotiques. Les parenthèses contenant le titre confirment ce silence autrefois imposé par la bienséance masculine:

Si escribiera un poema sobre nosotros, sería censurada.

Entonces, déjame contarte de cómo el junco es caña, y extiende su miel oculta adentro.

O de aquella orquídea violeta de pétalos y pétalos que navegan en savia, o de cómo se abre y penetran estrellas que iluminan la sangre, aquella que dormía. Y de cómo unos ojos se beben entero el diccionario.

Pero, hagamos un trato, no contemos a nadie, para que este poema no muera censurado (56).

Las palabras del cuerpo (2001) se donne comme objectif d'offrir la possibilité au corps de la femme de prendre la parole et d'exprimer sa réalité, ses sensations, ses désirs, ses abandons et aussi ses mystères. La voix poématique le notifie expressément dans les deux vers suivants qui peuvent faire figure de manifeste littéraire:

Escribo con mi lengua

El poema de mi cuerpo. («No escucharé...», 34)

$\mathrm{Au}$ cours de cet article, je souhaiterais vous démontrer comment, au travers d'une étonnante érotisation poétique de son corps, la voix poématique espinolienne fait de son corps de femme - de son «corps-poursoi» (Sartre 1943: 372-373) -, une fenêtre ouverte sur le monde sensible et sublime, une fenêtre ouverte sur le cosmos.

Vous l'aurez compris, Lourdes Espínola transgresse ici les tabous et les interdits que la société patriarcale ultra-catholique et la famille imposent trop souvent encore à la femme latino-américaine: elle entend parler en toute liberté de son corps et de ses plaisirs. L'œuvre de Lourdes Espínola marque ainsi une rupture par rapport à l'expression du désir et à l'affirmation du corps en poésie latino-américaine, expression qui jusque-là venait du côté des plumes masculines. Lourdes Espínola rompt donc avec la tradition et ose 
faire émerger un corps masculin érotisé, brut objet de désir charnel. Cette inversion répond à des exigences de survie physique et poétique.

$\mathrm{Au}$ niveau esthétique, Lourdes Espínola marque, dans ce recueil, un changement tout aussi provocateur: Las palabras del cuerpo se compose de 54 pièces courtes (le plus long poème, "La pipa», ne comporte en effet que 27 vers) parmi lesquelles seuls 17 poèmes proposent un titre ${ }^{1}$, quelquesuns s'ouvrent par ailleurs sur une dédicace ou une citation ${ }^{2}$. Construites en alternant vers d'art mineur et vers d'art majeur sans logique apparente, ces pièces ne présentent dans tout le recueil qu'une dizaine de rimes, dues selon nous plus au hasard des modulations poétiques qu'à un effet de style ${ }^{3}$. La liberté est donc ce qui semble motiver l'expression du sujet lyrique.

Élément tellurique, le corps de la voix espinolienne est d'abord une terre trop sèche, parfois aride. Le premier poème de Palabras del cuerpo reprend bien cette géographie du corps en mettant en évidence différentes parties du corps reliées entre elles par quelques cours d'eau. La carte physique du corps espinolien est donc la suivante:

Existe un mapa de antiguas cicatrices:

en un seno que va directo al corazón,

un camino que zigzaguea hasta mi sexo.

Obviemos los valles y las colinas,

están los territorios: secos y húmedos

senderos a otra parte. («Mi cuerpo», 10)

En partie inexploré, ce vaste corps mystérieux ( «Mi cuerpo es el misterio universal / la geografía casi inexplorada», 10) est également corps perméable, coquille poreuse, attirée par la lumière et la musicalité d'un autre corps masculin, sa moitié, faisant office de boussole («argonauta de la rota brújula», 10). Dans ce recueil coexistent donc deux corps structurés par un jeu infini de miroirs; un corps permettant à l'autre d'exister grâce au reflet qu'il lui propose:

1 «Nombre» (18), «Cuerpo» (26), «De cómo escriben las mujeres poemas eróticos» (56), «El revés de la trama» (58), «A mi cuerpo » (64), «Cuerpo » (66), «Mito de creación » (68), «Espejo» (73), «El revés del tapiz» (78), «En el terreno de lo sagrado» (80), «Destino de poesía (92), «My sole desire» (94), «Mis hermanos indígenas» (98), «¿Quién me dirá si en el secreto archivo de Dios están las letras de mi nombre?» (104), «La pipa» (106), «Naciendo» (108) et «Teléfono» (116).

2 «Mira hijo...» (22) et «Amado, no pienso en ti...» (48).

3 Ces rimes se retrouvent aspirées à chacune de leur apparition par un enjambement, devenant ainsi rimes internes. 


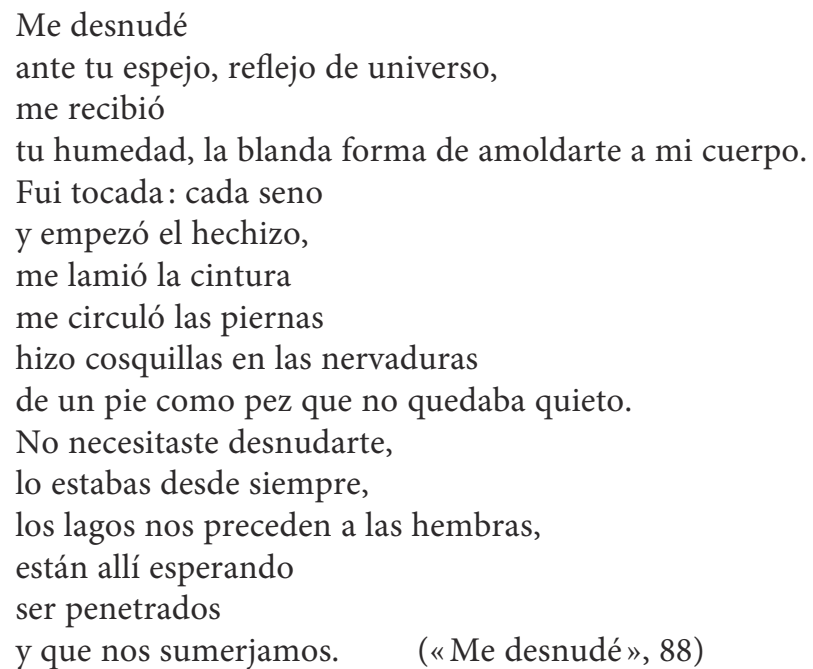

Même s'il est question de s'affirmer par rapport à l'homme, la voix poématique féminine comprend dès la deuxième pièce de notre recueil qu'elle ne pourra guère composer sans la voix et le corps de l'homme (ou son reflet). Créature en voie d'extinction - parce que trop humaine -, elle est attirée par ce corps masculin corps-lumière, corps-couleur et corps-sons. Las palabras del cuerpo nous propose un réseau cohérent construit autour des adjectifs possessifs (des première et deuxième personnes du singulier) délimitant à l'intérieur du corps du poème l'espace réservé au corps féminin et au corps masculin. Ce jeu des possessifs dessine la géo-poésie de ces deux corps : leur territoire respectif serait un cercle qui, lorsqu'il rencontre l'autre, forme une spirale ascensionnelle.

Le toucher joue un rôle fondamental dans la rencontre de ces deux corps : en effet, à lui seul, il est capable d'engendrer dans la voix poématique espinolienne toutes les facultés humaines (mémoire, imagination, entendement, désir, volonté...). Les caresses, par exemple, sont indispensables à la genèse et la découverte du corps propre espinolien et permettent de faire le départ entre l'impression ressentie et la réalité extérieure à laquelle ces caresses renvoient. En faisant du toucher (et non de la vue) le vecteur de l'idée d'étendue, Lourdes Espínola reprend une tradition qui fait la part belle à ce que Lucrèce considérait comme le «sens suprême».

Différentes étapes marquent en outre l'émergence du corps propre de la voix poématique espinolienne et définissent la progressive rencontre avec l'Autre masculin: par l'appréhension d'une contiguïté, elle peut apercevoir l'étendue que représente son corps; il faut donc que la main (celle de 
l'homme ou la sienne) s'aventure activement et que le toucher s'accompagne de mouvement. Aussi paradoxal que cela puisse paraître, le toucher donne la distance, l'extériorité. Le corps espinolien doit toucher et se toucher afin de distinguer les corps extérieurs à son propre corps. En se touchant, la voix poématique espinolienne commence par se découvrir plusieurs: elle est ici, là, ailleurs. Elle fait progressivement émerger son corps pour que celui-ci ne reste pas éclaté et que sa géographie soit progressivement explorée par le corps masculin:

Primero fue tu mano, como un pez deslizado, en la humedad de mis piernas. Luego el estilete de tu lengua en mi boca, en la humedad de mi deseo. Y la sacra ofrenda entre tus manos... posada en vedados territorios. («En el terreno de lo sagrado», 80)

Ces caresses sont accompagnées d'un sentiment qui confère au corps espinolien le privilège d'être aussi vécu. En outre, ces caresses, réclamées par la voix poématique, ouvrent le corps espinolien sur une lumière vitale pour lui :

Me desnudé entera:

de los dedos al vientre,

de la piel hasta ti,

de mi latido a tu mano.

Me extendí,

la ofrenda de los dioses:

palpitante, tibia,

balbuciente en secretos.

Y pusiste tus manos

ahuecadas en nidos,

y sentiste los fuegos

y cerraste los ojos.

La luz brillante ciega cuando no la esperamos. («Me desnudé...», 74)

Bien entendu, seul l'amour physique - caresse ultime - permettra à ces deux cercles, non de fusionner, mais de «converger» et de devenir cercles cosmiques:

En algún punto

los círculos concéntricos

se tocaron: 
extraños meteoros

de rápidos planetas. («Pueden pasar dos cosas...», 36)

Pour nous en convaincre, lisons également le court poème suivant: repérons-y le jeu des possessifs et centrons-nous au dernier vers sur ce verbe-clef, « converger»:

Tengo guardado, entero,

el deseo infinito de tu cuerpo

ligado entre mis piernas,

los secretos pasajes interiores.

Tengo memorias de círculos

sintiendo dentro mío

la explosión luminosa de tu savia:

tu volcán convergido entre mis labios. ( TTengo guardado...», 28)

Nous le comprenons aisément: la voix poématique a tout intérêt à se rapprocher de cet autre, être de lumière et de sons, afin de l'embrasser, d'englober cet autre qui, tel un miroir, renverrait au corps espinolien, son image la plus parfaite. Face à face, ces deux corps deviennent alors «dos amantes-circulares / desnudos-infinitos». Les traits d'union que nous retrouvons dans les deux vers cités, si rares en langue espagnole, cristallisent icil'union parfaite de ces deux corps qui se réfléchissent et parfois s'englobent, tels des corps-gigognes cosmiques ( $«$ mis poemas prendidos de tu mano / mi piel prendida de tus ojos, / mis piernas trepadas a tus muslos. / Un abrazo anidando mis senos/y mis manos perdidas en tu nuca./ Mi lengua sin barreras, / y la tuya, pequeño sable-beso, / mi corazón, / un cascarón / donde se esconde el tuyo", in "Débiles hombres...», 12).

Lorsque la spirale de ces deux corps est parfaite, que le reflet de l'un se confond avec le reflet de l'autre, les possessifs de première et deuxième personne du singulier se frôlent également et finissent par se fondre dans des pronoms sujets et adjectifs possessifs de première personne du pluriel, donnant naissance à une nouvelle modulation d'une voix poématique désormais gynandre:

Nuestros cuerpos mojados por el agua,

lamidos, enredados,

el mar cosquillea nuestro sexo,

un pie, la nuca...

El agua que espumosa

nos agarra, un brazo

como roce al descuido

y te atrapa, 
me atrapa...

pero fluyo, te extiendes.

Nuestras alas se rozan,

nuestras piernas se anudan,

y el agua nos hamaca:

de dentro, afuera y dentro.

Nuestros cuerpos: el agua. («Nuestros cuerpos...», 14)

Ou encore:

Estabas dentro mío

como sable de seda

explorando una flor

y todos mis sentidos

convergieron en ese epicentro:

corazón-latido-vida.

«Mírate al espejo»

me dijiste,

$\mathrm{y}$ fui testigo

de dos amantes-circulares

desnudos-infinitos...

Completos caracoles con rugidos de mar.

¿Dónde empecé, dónde empezabas?

Éramos, nuevamente

el anillo de fuego de los tiempos,

Por eso,

regalo de la imagen,

hoy amo los espejos. («Espejo », 72)

Comme le suggère la seule occurrence d'adjectifs possessifs de la première personne du pluriel de tout le recueil, répété ici cinq fois, et comme le laisse deviner l'ouverture au pluriel entre le premier et le troisième poème (on est en effet passé de «mi cuerpo » à «nuestros cuerpos»), le corps masculin habite le corps de la voix poématique espinolienne qui, lui, se révèle tout imprégné de la sonorité, des couleurs et des odeurs de l'Autre masculin:

Se instalan tus olores, tu sonrisa,

tu cara brillante y ladeada

También tus ojos - a veces tan temibles y sabios -

brillan constelaciones de palabras

y sonidos que paralizan mi memoria. («Amado, no pienso en ti...», 48)

Si ces deux corps ne font a priori que se frôler, la lecture de ce deuxième poème nous confirme néanmoins leur dissolution physique au cours de l'acte amoureux puisque ces deux corps deviennent eau: «Nuestros cuerpos: 
el agua». Contre toute attente, le corps masculin sera le révélateur sonore et lumineux du corps de la voix poématique: en s'imbriquant en elle, il fera de ce corps / de leur corps une caisse de résonance du plaisir («caja de resonancia del placer», "jaula de piel, pelo y sonido", 64) et fera du corps espinolien une intériorité ouverte, un corps-cosmos:

Me desnudé

ante tu espejo, reflejo de universo,

me recibió

tu humedad, la blanda forma de amoldarte a mi cuerpo.

(«Me desnudé...», 88)

Dans ce nouveau corps-cosmos fruit du plaisir et de la jouissance, l'esprit de la voix poématique s'incarne dans une chair qui est à la fois celle du sujet, celle du monde et celle des mots. La nudité de la voix poématique (qui expliquerait l'absence de toute parure poétique comme la rime) traduit un état du corps exemplairement ouvert à l'autre et au monde, offert non pas au viol du regard, comme l'est trop souvent le nu, mais à la douceur des caresses et à la subtilité du toucher:

$\mathrm{Tu}$ caricia es un lazo, seda atada a mi piel, terciopelo que se extiende y se amarra entre mis piernas.

[...]

Tu caricia desata el alocado mar de mis gemidos. («Tu caricia...», 42)

Le corps de l'homme permet au corps de la voix poématique espinolienne de s'ouvrir au monde et d'exister. Le corps de la voix poématique apparaît ainsi comme une sorte d'échangeur entre la conscience et le monde extérieur. Tel qu'elle le vit «de l'intérieur», son corps ne se distingue pas aussi nettement du monde extérieur; il en reçoit les messages et renvoie la réponse au-dehors. Elle n'y est pas enfermée, puisqu'elle est ouverte, grâce au corps masculin, sur le monde et qu'il lui permet de s'y mouvoir; le corpscosmos espinolien est lui-même l'organe de cette circulation incessante et à double sens du dehors au-dedans et vice-versa, qui est la condition de la vie et de la parole, et dont la respiration est la manifestation emblématique, intimement liée au rythme du poème:

Una palabra que contiene el mundo, el crepúsculo en mi seno a dos colores el volcán convergiendo entre mis piernas 
y el espacio callado de tus labios.

El lazo de tu sexo se desata

rojo de seda como candelabro.

Entras en eclosión a mi universo:

en la explosión más violenta,

la más dulce... («Una palabra...», 114)

Le corps espinolien voit en lui la traduction, à un niveau inférieur de l'harmonie du monde. Lourdes Espínola reprend à son compte l'idée médiévale d'une harmonie entre le corps humain et le cosmos, mais lui donne un développement et une concrétude dont elle était privée au Moyen Âge - où elle avait un sens métaphysique, mais restait encore disjointe des représentations artistiques. Ici, en revanche, c'est dans le corps même du poème que s'incarne cette correspondance du corps de la voix poématique et du monde, du microcosme et du macrocosme. Le monde est un enroulement sur soi puisque chaque chose le composant est ce qu'elle est en vertu d'une ressemblance avec une autre. Michel Foucault avançait, dans Les Mots et les Choses, l'idée suivante:

De loin le visage est l'émule du ciel, et tout comme l'intellect de l'homme reflète, imparfaitement, la sagesse de Dieu, de même les deux yeux, avec leur clarté bornée, réfléchissent la grande illumination que répandent, dans le ciel, le soleil et la lune; la bouche est Vénus, puisque par elle passent les baisers et les paroles d'amour; le nez donne la minuscule image du sceptre de Jupiter et du caducée de Mercure. (34)

Loin de l'enfermer en soi-même et dans la forme graphique figée du poème, ce microcosme vivant, vibrant et sonore révèle l'appartenance de la voix poématique au macrocosme u-topique et sonore. Dans le corps, et dans ce symbole transversal de l'œuvre poétique espinolienne que sont les cicatrices, se trouve en quelque sorte écrite l'histoire de la voix poématique et de l'univers à la manière d'un palimpseste infini où se superposent les reliques de la chaîne infinie des corps. Palimpseste infini ou registre exhaustif du monde, chaque corps évoque en même temps les plis de son âme qui sont tout autant de perceptions aveugles, obscures, comme repliées sur elles-mêmes.

Loin d'être réservé à des états exceptionnels, l'échange entre le corps et le cosmos apparaît comme une loi élémentaire de la physiologie dans la trajectoire poétique de notre poétesse. Lourdes Espínola a su exprimer et analyser cette unité organique de la chair du monde. La science moderne révèle au poète que la totalité du monde vivant parle le même langage; si l'homme peut être ému jusqu'aux entrailles par le spectacle du monde, 
c'est qu'il est fait de la même chair que lui. Ce sentiment d'appartenance à la chair du monde se fonde sur la connaturalité du corps humain et de son environnement: nous restons des parties de la nature, des visages de son activité, des façons d'être de son énergie. Et cette solidarité de la matière vivante et de son propre corps, la voix poématique l'éprouve là où la nature semble la plus hostile ou la plus indifférente à l'homme. Cette communion corps et cosmos permet à la voix poématique de naître simultanément avec le monde et le poème: elle se retrouve incarnée dans la lettre même des mots ( «Las palabras del cuerpo»), auxquels le rythme et la musicalité rendent leur teneur concrète et leur capacité d'évoquer un corps et un cosmos en marche synchronisée.

Le corps de la voix poématique espinolienne est toujours «la moitié possible d'un atlas universel» (Foucault: 37-38), la somme historique de tous les mythes:

Convergen en mi cuerpo

los callados mitos.

Tu sexo, minotauro

y la creación de los gemelos en mis senos.

El árbol de la vida en mi vientre

y la sinfonía de todos los adioses.

Depósito de antiguas historias... («Cuerpo», 66)

Cette relation entre corps et monde a longtemps été assurée en poésie par un système de correspondances symboliques, d'inspiration mythique ou théologique, qui faisait du microcosme un reflet du macrocosme. Créés par le même Verbe divin, l'un et l'autre étaient porteurs de signes que le poète avait pour tâche de déchiffrer ou d'interpréter, mais qu'il n'avait pas à produire ni même à éprouver. Cette universelle analogie ayant été remise en cause par la modernité, le corps s'est retrouvé dans une solitude et enfermé dans une contemplation fascinée de sa beauté déserte et désertée.

À cette pensée et à cette esthétique, s'opposent, vous l'aurez compris, la phénoménologie de Lourdes Espínola et sa poésie de l'incarnation, qui font du corps de la voix poématique le médiateur de notre relation avec le monde et avec l'Autre. Si la conscience espinolienne peut être définie comme "être au monde», c'est en un double sens: elle ne se borne pas à y être, elle en est partie prenante. Dans cette relation, «le corps est de la partie»; c'est «la corporéité de la conscience» qui fonde, pour Lourdes Espínola, sa "participation au monde qu'elle constitue» (Lévinas 1982: 156). Ce rapport du corps à lui-même "fait de lui le vinculum du moi et des choses" 
(Merleau-Ponty 1960: 212-213), le foyer d'un monde dont il fait partie: «Le corps propre est dans le monde comme le cœur dans l'organisme [...] il forme avec lui un système [...] La chose et le monde me sont donnés avec les parties de mon corps [...] dans une connexion vivante comparable ou plutôt identique à celle qui existe entre les parties de mon corps lui-même» (Merleau-Ponty 1945: 272). C'est ce que nous livre le poème suivant:

Abrí mis piernas

para dar luz al sol,

el calor derritiéndose en mi costado

y su luz

iluminando mis rodillas.

Y las articulaciones al girar

hicieron música

que se aquietaba en las horas.

Abrí los brazos

y de los senos brotó la luna

entonces tu lengua

me levantó hacia la humanidad entera.

(«Mito de creación », 52)

Dès lors, Lourdes Espínola peut dire indifféremment que «[son] corps est fait de la même chair que le monde» (Merleau-Ponty 1964: 303), et que «le monde est fait de l'étoffe même du corps» (Merleau-Ponty 1985: 18-19). C'est grâce à sa commune appartenance à une même chair, qui est celle du monde, que la voix poématique / le je espinolien peut communiquer avec le monde, l'embrasser et le rendre heureux:

No podré desentrañar el mundo, desnudar el secreto de sus voces, pero lo haré feliz.

Haré bailar estrellas con mi pluma, temblarán unas venas

con mi verbo...

La punta del poema

cosquilleará un borde desconocido, lejano, tal vez solo.

Y empezará a sacudirse su savia y mis palabras.

Y eso, también es un misterio. («Destino de poesía», 92)

Le corps-cosmos espinolien, fait de "chair du monde», éclaire ainsi d'un jour nouveau la correspondance traditionnelle en poésie entre macrocosme et microcosme. Elle permet de la fonder non plus sur une symbolique préétablie et sur une métaphysique, mais sur l'expérience sensible. Elle 
éclaire notamment l'érotisme cosmique qui se déploie souvent dans toute la poésie espinolienne.

Le corps espinolien cherche, comme le fit le je rimbaldien, à «sentir un peu [l]'immense corps» de l' «Être» en qui s'incarne la «beauté» du monde, et il sent ses propres "os" "revêtus d'un nouveau corps amoureux» (Rimbaud 1972: 140 et 127). C'est ce «nouvel amour», qui embrase les corps et les cœurs espinoliens, et qui embrasse le cosmos dans Las palabras del cuerpo.

Corps-cosmos en état d'hypnose "ouverte» et de réceptivité pure, sa voix / la voix du poème prend une valeur moins d'expression que de signe: les mots fortuits qui montent à la surface de l'esprit, le refrain, l'obsession d'une phrase forment une espèce d'incantation qui finit par coaguler la conscience. Le corps-cosmos devient alors, comme chez Claudel, un instrument privilégié de la "co-naissance au monde et de soi-même».

Cette recherche s'opère elle aussi d'abord à tâtons au plus obscur de sa chair, dans les mille replis d'une matière ingénieuse, où des messages se mettent à circuler, frayant des chemins imprévus à sa pensée:

Una acuosa lengua

que explora y se deshoja,

se esconde y se desata

y que habla violenta en el silencio. («Mi cuerpo...», 10)

Ou encore:

Vi que el espejo de tu pupila

susurraba verdades

que me dejaron desgajada y desnuda:

con la piel en madeja.

Temblé con tu nombre. («Cuando mi pecho...», 16)

Dans la caverne de la gorge de la voix poématique, animée de secrets mouvements, commencent à vibrer les cordes vocales:

Al pronunciar tu nombre,

la redonda luna se me quedó en la lengua.

Las vocales de tu nombre

saltaron al lago de mi garganta.

Las consonantes fantasmales, bailarinas

luminosas y claras,

desde mi vientre te nombraron.

Lucharon las primitivas fuerzas.

Con mi voz fui caminando 
el peregrino sendero

hasta tu boca. («Nombre», 18)

Ou encore:

digo tu nombre, al borde de mi lengua.

Y rueda el sonido

como mi corazón:

cáscara impregnada de tus manos

que aún late al fin de tu llamada. （ TTeléfono», 116)

«La gesticulation émotionnelle par laquelle l'homme superpose au monde donné le monde selon l'homme» est, comme nous le rappelle Merleau-Ponty, une des premières ébauches du langage»; elle lui fournit le modèle à partir duquel il pense la parole, qui repose elle-même sur un comportement corporel: le mot, "avant d'être l'indice d'un concept, est d'abord un événement qui saisit le corps» (272). La pensée n'existe pas avant ni indépendamment du langage, elle ne prend corps qu'à travers sa formulation, et c'est tout particulièrement vrai du discours poétique, dans lequel le sens est indissociable de la forme même de l'expression. À la différence de la signification conceptuelle, transcendante et arbitraire, le signifié poétique entretient une relation immanente et nécessaire avec les signifiants: il s'incarne dans la chair même des mots, dans leur résonnance comme dans leur physionomie graphique. Il s'agit pour Lourdes Espínola d'un «sens émotionnel», qui résulte des qualités sensibles des mots et des choses: "les mots, les voyelles, les phonèmes" deviennent dans le poème «autant de manières de chanter le monde »; et "ils son destinés à représenter les objets, non pas comme le croyait la théorie naïve des onomatopées, en raison d'une ressemblance objective, mais parce qu'ils en extraient et au sens propre en expriment l'essence émotionnelle» (218).

Si le style est ce qui imprime dans l'écriture la marque de l'intime, les modernes ont tendance à en chercher la source du côté d'une idiosyncrasie physiologique autant que psychologique; «le style, c'est l'homme, et c'est son corps», disait Artaud (130). Et Roland Barthes définit le style comme «la voix décorative d'une chair inconnue et secrète»; il n'est «que le terme d'une métamorphose aveugle et obstinée, partie d'un infra-langage qui s'élabore à la limite de la chair et du monde»: "son secret est un souvenir enfermé dans le corps de l'écrivain» (1953: 20-21).

Toutes ces réflexions et ces recherches s'inscrivent dans le prolongement de la pratique de Lourdes Espínola elle-même. Renonçant au primat de la conception sur l'expression érigée en précepte par le classicisme, la poésie 
moderne confère une part d'initiative aux mots, et à leur qualité sensibles le pouvoir d'éveiller et de produire le sens au lieu de simplement le traduire, l'imiter ou l'orner. Ce n'est pas un hasard si cette libération de la femme et de la signifiance s'est accompagnée d'une émancipation du vocabulaire anatomique et d'une promotion du corps comme métaphore privilégiée du langage poétique. Cette "physique» du langage aura permis à notre poétesse d'écrire les mots du corps enfin délivrés, enfin autonomes, enfin capables de donner naissance et corps à la signification.

Organisme dépositaire de la vie, le Mot présente, dans ses voyelles et ses diphtongues, comme une chair; et, dans ses consonnes, comme "une architecture délicate» (Mallarmé 1998: 949). Non seulement la poésie est indissociable de la matérialité des vocables, mais elle a besoin du support de la voix, venue du fond du corps. En donnant au poème la densité d'un «corps verbal», et en favorisant «les rapprochements physiques des mots», Lourdes Espínola échappe à l'abstraction du concept pour restituer à la pensée tout son poids d'incarnation, en même temps que son ouverture au cosmos. Parce qu'il s'agit de poésie, parce qu'il s'agit de $\pi$ 'in du corps espinolien s'incarne dans la chair même des mots, dont on sent toute la pulpe à l'occasion de la lecture à haute voix de cette pièce où, sous la voûte de son palais, déjà résonne le chant poétique. Sa peau / son corps é-mu et ex-cité est devenu le lieu d'un échange intense et incessant entre le dedans et le dehors, les idées et les sensations, les mots et l'émotion:

Mi cuerpo será el viejo chamán sabio

que siempre fue,

papel en blanco

al borde de escrituras

$\mathrm{y}$ virgen, siempre virgen.

Creando

el día primero,

escribo con mi lengua

el poema de mi cuerpo,

aquel que brota luz

y se rompe en estrellas,

cuando toma la pluma,

cuando tuerzo las piernas. («No escucharé...», 34)

Le corps devient en quelque sorte la page blanche sur laquelle la voix poématique se sonorise et sonorise à la fois l'expérience vécue et la réflexion qu'elle inspire. Lisons l'une des rares pièces comportant un titre et intitulée donc «Destino de poesía » (92): 
No podré desentrañar el mundo, desnudar el secreto de sus voces, pero lo haré feliz.

Haré bailar estrellas con mi pluma, temblarán unas venas

con mi verbo...

La punta del poema

cosquilleará un borde desconocido,

lejano, tal vez solo.

Y empezará a sacudirse

su savia y mis palabras.

Y eso, también es un misterio. («Destino de poesía», 92)

Le corps masculin apporte donc lumière, couleurs et sonorités au corps espinolien. Il lui permet enfin de reproduire l'harmonie cosmique:

Abrí mis piernas

para dar luz al sol,

el calor derritiéndose en mi costado

y su luz

iluminando mis rodillas.

Y las articulaciones al girar

hicieron música

que se aquietaba en las horas.

Abrí los brazos

y de los senos brotó la luna

entonces tu lengua

me levantó hacia la humanidad entera. ( Mito de creación», 68)

Le corps espinolien est un carrefour où se rencontrent le moi, le monde et les mots; comme tel, il est une source de poésie et une ressource pour la pensée et confirme bien la place centrale occupée par le corps dans la poétique et la philosophie contemporaines. La poésie est affaire de tact, et une poétique de l'incarnation revêt, dans une société qui idolâtre le corps mais souvent le pressure, le torture ou le prostitue, des enjeux qui ne sont pas seulement esthétiques, mais éthiques, voire politiques.

Le corps de la voix poématique espinolienne possède des sens particulièrement perméables aux sonorités masculines, mais aussi aux formes, aux couleurs. À partir de l'expérience sensorielle de la caresse, c'est un sens qui se découvre et s'élabore. Lourdes Espínola nous aura démontré, dans ce recueil, qu'elle avait relevé avec précision et justesse le défi que ce monde muet lance au langage, en redonnant du corps à celui-ci. Il s'agissait en quelque sorte de rendre à la langue son acception première d'organe 
de la parole: «la langue est un morceau de chair» (Prevel 1974: 5), aime à rappeler Noël, et il cherche à atteindre le point d'articulation de l'organique et du linguistique, «le lieu du corps où nous prenons langue» (Noël: 149). Le poème nous est donc offert ici comme un aperçu fugitif sur la vie intime du corps, arraché à la prison du code. Et de fait, par la force du langage et de l'image, Lourdes Espínola réussit à évoquer cet espace du dedans du corps, que peu de poètes s'étaient risqués à explorer, et qui devient, sous sa plume, une sorte de paysage cénesthésique où les frontières entre l'intérieur et l'extérieur se sont abolies.

\section{Bibliographie}

Artaud, Antonin, Euvres complètes, t. 21.

Barthes, Roland, 1953, Le Degré zéro de l'écriture, Paris, Le Seuil.

Dagonet F., 1993, La Peau découverte, Le Plessis-Robinson, Synthélabo, coll. «Les empêcheurs de penser en rond».

Espínola, Lourdes, 2001, Les mots du corps / Las palabras del cuerpo, présentation et traduction de Claude Couffon, Paris, Indigo et Côté-femmes édition.

Husserl, 1992, Méditations cartésiennes. Introduction à la phénoménologie, Paris, Vrin.

Levinas, Emmanuel, 1982, En découvrant l'existence avec Husserl et Heidegger, Paris, Vrin.

Joubeau, 1991, Le Corps humain dans la philosophie platonicienne. Étude à partir du Timée, Paris, Vrin.

Jaquet C., 2001, Le Corps, Paris, PUF, coll. Philosopher.

Mallarmé, Stéphane, 1998, «Les Mots anglais», in Euvres complètes, t.1, Paris, Gallimard, «La Bibliothèque de la Pléiade».

Merleau-Ponty, Maurice, 1945, Phénoménologie de la perception, Paris, Gallimard.

Merleau-Ponty, Maurice, 1960, "Le Philosophe et son ombre», in Signes, Paris, Gallimard.

Merleau-Ponty, Maurice, 1964, Le Visible et l'Invisible, Paris, Gallimard.

Merleau-Ponty, Maurice, 1985, L'œil et l'Esprit, Gallimard, Folio-Essais.

Noël, Bernard, 1975, Treize cases du je, Paris, Flammarion.

Prevel, Jacques, 1974, En compagnie d'Artaud, Paris, Flammarion.

Rimbaud, Arthur, 1972, «Aube», et «Being Beauteous», Illuminations in Cuvres complètes, Paris, Gallimard, «La Bibliothèque de la Pléiade».

Sartre, Jean-Paul, 1943, L'Être et le Néant, Essai d'ontologie phénoménologique, chap. II, $3^{\mathrm{e}}$ Gallimard, Bibliothèque de la Pléiade.

Valéry, 1957, «Réflexions simples sur le corps», Études philosophiques, Variété, Euvres, I, Paris. 